\title{
Economic Burden of Pneumococcal Disease in Individuals Aged 15 Years and Older in the Liguria Region of Italy
}

\author{
Matteo Astengo ${ }^{1, *(\mathbb{D})}$, Chiara Paganino ${ }^{1}$, Daniela Amicizia ${ }^{1}\left(\mathbb{D}\right.$, Cecilia Trucchi $\left.{ }^{1,2}{ }^{(}\right)$, Federico Tassinari ${ }^{1}(\mathbb{D}$, \\ Camilla Sticchi ${ }^{1}$, Laura Sticchi ${ }^{2}$, Andrea Orsi ${ }^{2}{ }^{\mathbb{D}}$, Giancarlo Icardi ${ }^{2} \mathbb{D}$, Maria Francesca Piazza ${ }^{1} \mathbb{D}$, \\ Bruno Di Silverio ${ }^{1}$, Arijita Deb ${ }^{3}$, Francesca Senese ${ }^{4}$, Gian Marco Prandi ${ }^{4}$ and Filippo Ansaldi 1,2 \\ 1 Regional Health Agency of Liguria (ALiSa), 16121 Genoa, Italy; Chiara.paganino@regione.liguria.it (C.P.); \\ Daniela.amicizia@unige.it (D.A.); Cecilia.trucchi@regione.liguria.it (C.T.); fedetassi@ymail.com (F.T.); \\ Camilla.sticchi@regione.liguria.it (C.S.); mariafrancesca.piazza@regione.liguria.it (M.F.P.); \\ bruno.disilverio@regione.liguria.it (B.D.S.); filippo.ansaldi@unige.it (F.A.) \\ 2 Department of Health Sciences (DiSSal), University of Genoa, 16132 Genoa, Italy; Laura.sticchi@unige.it (L.S.); \\ Andrea.orsi@unige.it (A.O.); icardi@unige.it (G.I.) \\ 3 Merck \& Co., Inc., Kenilworth, NJ 07033, USA; arijitadeb88@gmail.com \\ 4 MSD Italy, 00189 Rome, Italy; Francesca.senese@merck.com (F.S.); gian.marco.prandi@merck.com (G.M.P.) \\ * Correspondence: Matteo.astengo@regione.liguria.it; Tel.: +39-0105488257
}

\section{check for} updates

Citation: Astengo, M.; Paganino, C.; Amicizia, D.; Trucchi, C.; Tassinari, F.; Sticchi, C.; Sticchi, L.; Orsi, A.; Icardi, G.; Piazza, M.F.; et al. Economic Burden of Pneumococcal Disease in Individuals Aged 15 Years and Older in the Liguria Region of Italy. Vaccines 2021, 9, 1380. https://doi.org/ $10.3390 /$ vaccines 9121380

Academic Editors: Maarten J. Postma and Cornelis Boersma

Received: 19 October 2021

Accepted: 20 November 2021

Published: 24 November 2021

Publisher's Note: MDPI stays neutral with regard to jurisdictional claims in published maps and institutional affiliations.

Copyright: (C) 2021 by the authors Licensee MDPI, Basel, Switzerland. This article is an open access article distributed under the terms and conditions of the Creative Commons Attribution (CC BY) license (https:// creativecommons.org/licenses/by/ $4.0 /)$.
Abstract: Despite the availability of vaccines against Streptococcus pneumoniae, the global incidence and economic cost of pneumococcal disease (PD) among adults is still high. This retrospective cohort analysis estimated the cost of emergency department (ED) visits/hospitalizations associated with non-invasive pneumonia and invasive pneumococcal disease among individuals $\geq 15$ years of age in the Liguria region of Italy during 2012-2018. Data from the Liguria Region Administrative Health Databases and the Ligurian Chronic Condition Data Warehouse were used, including hospital admission date, length of stay, discharge date, outpatient visits, and laboratory/imaging procedures. $A \geq 30$-day gap between two events defined a new episode, and patients with $\geq 1$ ED or inpatient claim for PD were identified. The total mean annual number of hospitalizations for PD was 13,450, costing $\sim 49$ million per year. Pneumonia accounted for the majority of hospitalization costs. The median annual cost of hospitalization for all-cause pneumonia was $€ 38,416,440$ (per-capita cost: $€ 26.78$ ) and was $€ 30,353,928$ (per-capita cost: $€ 20.88$ ) for pneumococcal and unspecified pneumonia. The total number and associated costs of ED visits/hospitalizations generally increased over the study period. PD still incurs high economic costs in adults in the Liguria region of Italy.

Keywords: pneumonia; pneumococcal disease; pneumococcal infections; health resources; comorbidity; healthcare costs; economic burden

\section{Introduction}

Pneumococcal disease (PD) is caused by Streptococcus pneumoniae and includes invasive (e.g., meningitis, bacteremia, sepsis, and bacteremic pneumonia) and non-invasive (e.g., sinusitis, bronchitis, and non-bacteremic pneumonia) diseases [1,2].

Invasive disease occurs when pneumococcus enters sterile sites, such as blood or cerebrospinal fluid, causing very serious conditions, such as bacteraemic pneumonia, meningitis, and sepsis. Infection from the lungs may spread to surrounding tissues, resulting in pleurisy or pericarditis. Furthermore, pneumococcus can pass from the airways to the blood, resulting in septicemia. Through the blood, the pneumococcus can reach any organ, including the meninges (meningitis), the joints (arthritis, osteomyelitis), and the peritoneum (peritonitis) [1,2].

Invasive diseases are serious clinical forms that can be life threatening. Pneumococcus is estimated to cause approximately 1.6 million deaths worldwide each year.

Specifically, overall mortality from pneumococcal bacteremia ranges between $15 \%$ and $20 \%$ in the antibiotic era. Pneumococcal endocarditis is rare but usually affects one or both 
left-sided valves (more often on the aortic than the mitral valve) and causes high mortality rates, ranging from $28 \%$ to $60 \%$. Surgery may be required before a course of antibiotics is completed. Osler described the clinical triad of pneumococcal endocarditis, meningitis, and pneumonia (AKA Austrian's syndrome) in 1881. The triad is now infrequent, but it still occurs.

Despite the availability of pneumococcal conjugate vaccines (PCVs) and a 23-valent pneumococcal polysaccharide vaccine (PPSV23) as preventative interventions, PD continues to cause significant morbidity and mortality worldwide [3,4], with severe PD in particular reported to be associated with high medical costs [5].

Nasopharyngeal colonization by S. pneumoniae leads to cross-transmission, infection, and disease, and it is a predominant source of transmission of the bacterium from children to adults. Therefore, the vaccination of pediatric populations can reduce the transmission of S. pneumoniae, providing indirect protection to adults [6-8]. It has been shown that a reduction in the rates of vaccine-serotype invasive PD (IPD) among vaccinated children is accompanied by a decrease in the rates among adults, potentially due to the reduction of vaccine-serotype colonization in both age groups [8].

To address the disease and economic burden of PD, a 7-valent PCV (PCV7) was licensed in the European Union (EU) in 2000 for use among infants and young children [9]. In 2003, a large-scale vaccination program against S. pneumoniae started in the Liguria region of Italy, an administrative region of more than 1.6 million inhabitants [10-12]. In summer 2010, PCV7 was replaced by a 13-valent PCV (PCV13) in the regional vaccination plan, and it has been widely administered since autumn 2010 [11]. Regarding pneumococcal vaccination in adults, Liguria started a large-scale program of vaccination in 2000 with PPV23 in all subjects over 64 years of age and in high-risk groups, in particular in those at major risk of acquiring pneumococcal infection and complications. Cumulative coverage rates, although not available for the Liguria region, showed in a region bordering Liguria in 2007 a PPV23 uptake that ranged from $26 \%$ to $31 \%$ in the elderly, with an approximate value of about $23 \%$ in adults with underlying risk conditions [11]. Since 2013, Liguria has implemented a new recommendation for pneumococcal immunization in adults, offering PCV13 and PPV23 in series in subjects aged between 70 and 75 years, and in individuals with specific comorbidities that are considered to place them at an increased risk of pneumococcal diseases, regardless of age.

Infants in Liguria are eligible to receive PCV13 at the ages of three, five, and 11 months $[10,13]$. Vaccine coverage rates in Ligurian infants have been more than $90 \%$ since 2007 [14-16]. However, vaccine coverage rates in older adults were reported to be suboptimal ( 23\%) [11].

Underestimation of the burden of PD is a well-known phenomenon $[17,18]$. Therefore, to demonstrate the potential value of higher-valent vaccines, it is important to quantify the residual and vaccine-preventable burden of invasive and non-invasive PD following the introduction of PCV7 and PCV13 into the childhood immunization schedule. Although data on the burden of PD in Italy are available, some measures of burden stratified by age and risk factors for severe outcomes, such as hospitalizations and emergency department (ED) visits, are lacking $[19,20]$. In addition, there is little information about direct costs among individuals with PD in Italy. These data are key for implementing preventative strategies and efficient healthcare pathways.

The aim of this retrospective cohort analysis was to estimate the cost of ED visits and hospitalizations associated with all-cause, pneumococcal, and unspecified pneumonia, bacteremia, meningitis, and acute otitis media (AOM) among individuals $\geq 15$ years of age in the Liguria region of Italy during 2012-2018.

\section{Materials and Methods}

\subsection{Study Design and Population}

This was a retrospective observational cohort study to assess the epidemiologic and economic burden of all-cause pneumonia, unspecified or pneumococcal pneumonia, IPD, 
and AOM in Ligurian individuals aged $\geq 15$ years (average population $\geq 15$ years: $1,475,242$; average total population: 1,592,058) during the period between October 2012 to September 2018.

Patients with one or more inpatient or ED claims for PD and all-cause pneumonia (based on International Classification of Diseases, Ninth Revision, Clinical Modification [ICD-9-CM] diagnosis codes; Supplemental Table S1) were identified during the study period. An interval of $\geq 30$ days between two hospital visits, including back-to-back visits between 2 years, or in the ED defined a new episode for all case histories.

\subsection{Data Sources}

The study used data from the Liguria Region Administrative Health Databases and the Ligurian Chronic Condition Data Warehouse (CCDWH). The Ligurian Administrative Health Database stores healthcare data on the entire population of Liguria and includes data on all ED visits, outpatient visits, outpatient pharmaceutical dispensing records, and hospitalizations. The data collected include the date of visit or admission, information on diagnoses and procedures performed, length of stay, date of discharge, and discharge status (i.e., died or discharged alive). The CCDWH integrates four main routine administrative healthcare data flows belonging to the regional health service. The CCDWH records data gathered from multiple regional health service data sources (hospital discharge records (DRs), pharmaceutics, medical fee exemptions, outpatient visits, and laboratory/imaging procedures) within a specified period by means of a pre-defined algorithm based on the codes assigned to specific diagnoses and procedures [21]. Through a record-linkage system based on a civil registry database, residents' histories of healthcare events are constructed in order to depict the chronic condition of each patient. All data are archived in a relational database by means of big-data logic [21].

\subsection{Cost Inputs}

The cost of hospitalizations was based on diagnostic-related group costs for hospital admissions [22]. Cost estimates for hospitalizations and ED access, including admission for short-term observation, were determined from the perspective of the government as payer, and were based on data from the diagnosis-related group (DRG) system and procedures performed in the ED. In accordance with the regional reimbursement system, costs related to ED access followed by hospitalization were estimated based on the DRG system only. The number of pharmaceutical prescriptions, days of drug therapy use, and associated costs were recorded during a 6-month follow-up period.

In the economic impact analysis for pneumonia, IPD, or AOM possibly caused by S. pneumoniae, in subjects presenting with more than one diagnosis during hospitalization, the cost was attributed to the principal diagnosis present on the discharge form.

\subsection{Data Analyses}

ED access and hospitalizations for all-cause pneumonia and pneumococcal or unspecified pneumonia, AOM, and IPD were stratified by age group (15-64 years and $\geq 65$ years) and by underlying comorbidities.

For each outcome, the prevalence of each comorbidity was calculated per age group for the entire study period, and the $95 \%$ confidence interval (CI) range was estimated using the $\mathrm{z}$-value of normal distribution method. Data were analyzed using the JMP version 13.0.0 software (SAS Institute, Cary, NC, USA). Quantitative outcome variables were summarized as mean or median and standard deviation or interquartile range, and categorical variables as frequency distributions and $95 \%$ CIs.

The mean and per capita costs of ED access, including admission for short-term observation, whether followed by hospitalization or not, were estimated for the entire observation period. Finally, a linear regression was applied to assess trends in the total costs for ED access and hospitalizations for pneumococcal or unspecified pneumonia, IPD, and AOM during the study period. A value of $p<0.05$ was considered statistically significant. 


\section{Results}

\subsection{Patient Demographics}

In total, 91,654 records of patients $\geq 15$ years of age in the Liguria region were included in the study during the period 2012-2018. Demographics based on patient records by indication are presented in Table 1.

The prevalence of at least one comorbidity in the general Liguria population $\geq 15$ years of age during the study period was $24.47 \%$ (95\% CI: 24.40-24.54); cardiovascular disease in 8.45\% (95\% CI: 8.35-8.56) of patients, neoplasia in 5.91\% (95\% CI: 5.83-6.00), respiratory disease in $3.90 \%$ (95\% CI: 3.83-3.97), diabetes in 5.49\% (95\% CI: 5.40-5.57), and chronic renal failure in $1.26 \%$ (95\% CI: $1.22-1.30)$.

In the study population, $75.03 \%$ (95\% CI: 74.75-75.31) of patients had at least one comorbidity; cardiovascular disease in $48.83 \%$ (95\% CI: 48.5-49.15), neoplasia in $18.76 \%$ (95\% CI: 18.51-19.01), respiratory disease in $23.72 \%$ (95\% CI: $23.44-23.99)$, diabetes in $18.02 \%$ (95\% CI: 17.77-18.27), chronic renal failure in 15.01\% (95\% CI: 14.78-15.24), and other in $33.38 \%$ (95\% CI: 33.08-33.69).

\subsection{Total Number and Cost of ED Visits and Hospitalizations}

The median annual number of ED visits and hospitalizations as a result of pneumococcal and unspecified pneumonia (excluding all-cause pneumonia), IPD, and AOM was 13,450 , with a median annual cost of $€ 50.63$ million, in individuals aged $\geq 15$ years during 2012-2018. Total costs increased by 37\% over the study period ( $€ 41.15$ million in 2012 and $€ 56.50$ million in 2018), the cost per ED visit/hospitalization increased by $6 \%$ over the study period ( $€ 3454$ in 2012 and $€ 3671$ in 2018), and the annual cost per capita increased by $42 \%$ ( $€ 28.28$ in 2012 and $€ 40.17$ in 2018). Linear regression results showed a statistically significant upward trend in total costs for pneumococcal or unspecified pneumonia, IPD, and AOM during the study period $(p<0.0001)$.

\subsection{Number and Cost of ED Visits and Hospitalizations for Pneumonia, Meningitis, and Bacteremia}

The number and cost of ED visits and hospitalizations for all-cause pneumonia, pneumococcal and unspecified pneumonia, meningitis, and bacteremia are shown in Tables 2-5. Pneumonia accounted for the majority of hospitalization costs (Tables 2 and 3). The median annual cost of hospitalization for all-cause pneumonia and pneumococcal and unspecified pneumonia amounted to $€ 38,416,440$ (per-capita cost: $€ 26.78$ ) and $€ 30,353,928$ (per-capita cost: €20.88), respectively. Pneumococcal and unspecified pneumonia constituted $60 \%$ of total costs for hospitalization due to pneumococcal or unspecified diseases. Total access to ED visits and hospitalizations increased over the study period due to all-cause pneumonia (8324 in 2012 vs. 12,472 in 2018), pneumococcal-specific pneumonia (79 vs. 169), and unspecified pneumonia (6802 vs. 10,122) (Tables 2 and 3). There was also up to an approximately $50 \%$ increase in costs of ED visits and hospitalizations due to pneumonia during the study (Tables 2 and 3).

On the other hand, meningitis accounted for a relatively low number of ED visits and hospitalizations during the study period (Table 4). The number of ED visits and hospitalizations due to pneumococcal meningitis varied between 10 and 23 per year, costing between $€ 5529$ and $€ 13,016$ per event. For unspecified meningitis, there were between five and $11 \mathrm{ED}$ visits and hospitalizations per year, costing between $€ 6323$ and $€ 15,669$ per event. There were only two ED visits due to unspecified meningitis not leading to hospitalization, in 2013 and 2015, costing $€ 300$ and $€ 61$, respectively.

The number of cases and costs of bacteremia increased over the study period (Table 5). The number of ED visits and hospitalizations for pneumococcal bacteremia increased from 85 events in 2012 to 149 events in 2018. The cost per event also increased from $€ 6476$ to $€ 8395$ during the study period. A similar pattern was seen with unspecified bacteremia. Only six ED visits due to unspecified bacteremia not followed by hospitalization were recorded in patients $>64$ years of age, in 2012, 2017, and 2018, costing a total of $€ 1533$ during the study period. 
Table 1. Demographics of patient records.

\begin{tabular}{|c|c|c|c|c|c|c|c|c|c|c|}
\hline & \multirow{2}{*}{$\begin{array}{l}\text { All-Cause } \\
\text { Pneumonia } \\
(n=70,402)\end{array}$} & \multicolumn{2}{|c|}{$\begin{array}{l}\text { Pneumonia } \\
(n=61,559)\end{array}$} & \multicolumn{2}{|c|}{$\begin{array}{c}\text { Meningitis } \\
(n=192)\end{array}$} & \multicolumn{2}{|c|}{$\begin{array}{l}\text { Bacteremia } \\
(n=8587)\end{array}$} & \multirow{2}{*}{$\begin{array}{c}\text { Otitis Media } \\
(n=11,061)\end{array}$} & \multirow{2}{*}{$\begin{array}{l}\text { Other Invasive } \\
\text { Diseases * } \\
(n=7463)\end{array}$} & \multirow{2}{*}{$\begin{array}{c}\text { Total } \\
(n=91,654)\end{array}$} \\
\hline & & $\begin{array}{l}\text { Pneumococcal } \\
\quad(n=967)\end{array}$ & $\begin{array}{l}\text { Unspecified } \\
(n=60,592)\end{array}$ & $\begin{array}{l}\text { Pneumococcal } \\
\quad(n=129)\end{array}$ & $\begin{array}{l}\text { Unspecified } \\
\quad(n=63)\end{array}$ & $\begin{array}{l}\text { Pneumococcal } \\
\quad(n=121)\end{array}$ & $\begin{array}{l}\text { Unspecified } \\
(n=8466)\end{array}$ & & & \\
\hline Male, $n$; \% [95\% CI] & $\begin{array}{l}37,830 ; 53.73 \\
{[53.37-54.10]}\end{array}$ & $\begin{array}{c}513 ; 53.05 \\
{[49.90-56.19]}\end{array}$ & $\begin{array}{l}32,380 ; 53.43 \\
{[53.04-53.83]}\end{array}$ & $\begin{array}{c}63 ; 48.83 \\
{[40.21-57.46]}\end{array}$ & $\begin{array}{c}27 ; 42.85 \\
{[30.63-55.07]}\end{array}$ & $\begin{array}{c}65 ; 53.71 \\
{[44.83-62.60]}\end{array}$ & $\begin{array}{c}4440 ; 52.44 \\
{[51.38-53.50]}\end{array}$ & $\begin{array}{c}5640 ; 50.98 \\
{[50.05-51.92]}\end{array}$ & $\begin{array}{c}5640 ; 75.57 \\
{[74.6-76.55]}\end{array}$ & $\begin{array}{l}49,168 ; 53.65 \\
{[53.32-53.97]}\end{array}$ \\
\hline $\begin{array}{c}\text { Mean age (SD) } \\
\text { Age group, } n ; \% \\
{[95 \% \mathrm{CI}]}\end{array}$ & $72.8(18.76)$ & $73.20(16.63)$ & $75.23(16.83)$ & $62.55(14.89)$ & $63.29(17.82)$ & $70.53(16.06)$ & $75.69(14.72)$ & $46.58(18.39)$ & $66.26(15.92)$ & $69.56(20.28)$ \\
\hline 15-64 years & $\begin{array}{l}18,077 ; 25.68 \\
{[25.35-26.00]}\end{array}$ & $\begin{array}{c}272 ; 28.13 \\
{[25.29-30.96]}\end{array}$ & $\begin{array}{l}13,284 ; 21.92 \\
{[21.59-22.25]}\end{array}$ & $\begin{array}{c}63 ; 48.84 \\
{[40.21-57.46]}\end{array}$ & $\begin{array}{c}26 ; 41.27 \\
{[29.11-53.43]}\end{array}$ & $\begin{array}{c}50 ; 41.32 \\
{[32.55-50.10]}\end{array}$ & $\begin{array}{c}1709 ; 20.19 \\
{[19.33-21.04]}\end{array}$ & $\begin{array}{c}9077 ; 82.06 \\
{[81.35-82.78]}\end{array}$ & $\begin{array}{c}2202 ; 29.51 \\
{[28.47-30.54]}\end{array}$ & $\begin{array}{l}29,940 ; 32.67 \\
{[32.36-32.97]}\end{array}$ \\
\hline$\geq 65$ years & $\begin{array}{l}52,325 ; 74.32 \\
{[74.00-74.65]}\end{array}$ & $\begin{array}{c}695 ; 71.87 \\
{[69.04-74.71]}\end{array}$ & $\begin{array}{l}47,308 ; 78.08 \\
{[77.75-78.41]}\end{array}$ & $\begin{array}{c}66 ; 51.16 \\
{[42.54-59.79]}\end{array}$ & $\begin{array}{c}37 ; 58.73 \\
{[46.57-70.89]}\end{array}$ & $\begin{array}{c}71 ; 58.68 \\
{[49.90-67.45]}\end{array}$ & $\begin{array}{c}6757 ; 79.81 \\
{[78.96-80.67]}\end{array}$ & $\begin{array}{c}1984 ; 17.94 \\
{[17.22-18.65]}\end{array}$ & $\begin{array}{c}5261 ; 70.49 \\
{[69.46-71.53]}\end{array}$ & $\begin{array}{l}61,714 ; 67.33 \\
{[67.03-67.64]}\end{array}$ \\
\hline $\begin{array}{c}\text { Any comorbidity } n \\
\%[95 \% \mathrm{CI}]\end{array}$ & $\begin{array}{l}56,705 ; 80.54 \\
{[80.25-80.84]}\end{array}$ & $\begin{array}{c}786 ; 81.28 \\
{[78.82-83.74]}\end{array}$ & $\begin{array}{l}49,872 ; 82.31 \\
{[82.00-82.61]}\end{array}$ & $\begin{array}{c}87 ; 67.44 \\
{[59.36-75.53]}\end{array}$ & $\begin{array}{c}47 ; 74.60 \\
{[63.85-85.35]}\end{array}$ & $\begin{array}{c}93 ; 76.86 \\
{[69.35-84.37]}\end{array}$ & $\begin{array}{c}7614 ; 89.94 \\
{[89.30-90.58]}\end{array}$ & $\begin{array}{c}3168 ; 28.64 \\
{[27.80-29.48]}\end{array}$ & $\begin{array}{c}6215 ; 83.28 \\
{[82.43-84.12]}\end{array}$ & $\begin{array}{l}68,765 ; 75.03 \\
{[74.75-75.31]}\end{array}$ \\
\hline Chronic renal failure & $\begin{array}{l}11,386 ; 16.17 \\
{[15.90-16.44]}\end{array}$ & $\begin{array}{c}130 ; 13.44 \\
{[11.29-15.59]}\end{array}$ & $\begin{array}{l}10,508 ; 17.34 \\
{[17.04-17.64]}\end{array}$ & $\begin{array}{c}6 ; 4.65 \\
{[1.02-8.29]}\end{array}$ & $\begin{array}{c}6 ; 9.52 \\
{[2.28-16.77]}\end{array}$ & $\begin{array}{c}14 ; 11.57 \\
{[5.87-17.27]}\end{array}$ & $\begin{array}{c}2028 ; 23.95 \\
{[23.05-24.86]}\end{array}$ & $\begin{array}{c}122 ; 1.10 \\
{[0.91-1.30]}\end{array}$ & $\begin{array}{c}1322 ; 17.71 \\
{[16.85-18.58]}\end{array}$ & $\begin{array}{l}13,760 ; 15.01 \\
{[14.78-15.24]}\end{array}$ \\
\hline $\begin{array}{l}\text { Cardiovascular } \\
\text { disease }\end{array}$ & $\begin{array}{l}38,109 ; 54.13 \\
{[53.76-54.50]}\end{array}$ & $\begin{array}{c}469 ; 48.50 \\
{[45.35-51.65]}\end{array}$ & $\begin{array}{l}34,603 ; 57.11 \\
{[56.71-57.50]}\end{array}$ & $\begin{array}{c}36 ; 27.91 \\
{[20.17-35.65]}\end{array}$ & $\begin{array}{c}23 ; 36.51 \\
{[24.62-48.40]}\end{array}$ & $\begin{array}{c}45 ; 37.19 \\
{[28.58-45.80]}\end{array}$ & $\begin{array}{c}5079 ; 59.99 \\
{[58.95-61.04]}\end{array}$ & $\begin{array}{c}703 ; 6.36 \\
{[5.90-6.81]}\end{array}$ & $\begin{array}{c}4359 ; 58.41 \\
{[57.29-59.53]}\end{array}$ & $\begin{array}{l}44,752 ; 48.83 \\
{[48.5-49.15]}\end{array}$ \\
\hline Respiratory disease & $\begin{array}{l}19,734 ; 28.03 \\
{[27.70-28.36]}\end{array}$ & $\begin{array}{c}255 ; 26.37 \\
{[23.59-29.15]}\end{array}$ & $\begin{array}{l}16,599 ; 27.39 \\
{[27.04-27.75]}\end{array}$ & $\begin{array}{c}10 ; 7.75 \\
{[3.14-12.37]}\end{array}$ & $\begin{array}{c}4 ; 6.35 \\
{[0.33-12.37]}\end{array}$ & $\begin{array}{c}19 ; 15.70 \\
{[9.22-22.19]}\end{array}$ & $\begin{array}{c}1441 ; 17.02 \\
{[16.22-17.82]}\end{array}$ & $\begin{array}{c}510 ; 4.61 \\
{[4.22-5.00]}\end{array}$ & $\begin{array}{c}1460 ; 19.56 \\
{[18.66-20.46]}\end{array}$ & $\begin{array}{l}21,738 ; 23.72 \\
{[23.44-23.99]}\end{array}$ \\
\hline Diabetes & $\begin{array}{l}13,408 ; 19.04 \\
{[18.75-19.33]}\end{array}$ & $\begin{array}{c}184 ; 19.03 \\
{[16.55-21.50]}\end{array}$ & $\begin{array}{l}11,686 ; 19.29 \\
{[18.97-19.60]}\end{array}$ & $\begin{array}{c}16 ; 12.40 \\
{[6.71-18.09]}\end{array}$ & $\begin{array}{c}6 ; 9.52 \\
{[2.28-16.77]}\end{array}$ & $\begin{array}{c}15 ; 12.40 \\
{[6.52-18.27]}\end{array}$ & $\begin{array}{c}2040 ; 24.09 \\
{[23.19-25.01]}\end{array}$ & $\begin{array}{c}531 ; 4.80 \\
{[4.40-5.20]}\end{array}$ & $\begin{array}{c}1647 ; 22.07 \\
{[21.13-23.01]}\end{array}$ & $\begin{array}{l}16,516 ; 18.02 \\
{[17.77-18.27]}\end{array}$ \\
\hline Neoplasia & $\begin{array}{l}13,759 ; 19.54 \\
{[19.25-19.84]}\end{array}$ & $\begin{array}{c}204 ; 21.10 \\
{[18.52-23.67]}\end{array}$ & $\begin{array}{l}12,096 ; 19.96 \\
{[19.64-20.28]}\end{array}$ & $\begin{array}{c}20 ; 15.50 \\
{[9.26-21.75]}\end{array}$ & $\begin{array}{c}13 ; 20.63 \\
{[10.64-30.63]}\end{array}$ & $\begin{array}{c}28 ; 23.14 \\
{[15.63-30.65]}\end{array}$ & $\begin{array}{c}2739 ; 32.35 \\
{[31.36-33.35]}\end{array}$ & $\begin{array}{c}538 ; 4.86 \\
{[4.46-5.26]}\end{array}$ & $\begin{array}{c}1433 ; 19.2 \\
{[18.31-20.1]}\end{array}$ & $\begin{array}{l}17,195 ; 18.76 \\
{[18.51-19.01]}\end{array}$ \\
\hline Others & $\begin{array}{l}25,517 ; 36.24 \\
{[35.89-36.60]}\end{array}$ & $\begin{array}{c}370 ; 38.26 \\
{[35.20-41.33]}\end{array}$ & $\begin{array}{l}22,611 ; 37.32 \\
{[36.93-37.70]}\end{array}$ & $\begin{array}{c}31 ; 24.03 \\
{[16.66-31.40]}\end{array}$ & $\begin{array}{c}16 ; 25.40 \\
{[14.65-36.15]}\end{array}$ & $\begin{array}{c}39 ; 32.23 \\
{[23.90-40.56]}\end{array}$ & $\begin{array}{c}3770 ; 44.53 \\
{[43.47-45.59]}\end{array}$ & $\begin{array}{c}1029 ; 9.30 \\
{[8.76-9.84]}\end{array}$ & $\begin{array}{c}2429 ; 32.55 \\
{[31.48-33.61]}\end{array}$ & $\begin{array}{l}30,595 ; 33.38 \\
{[33.08-33.69]}\end{array}$ \\
\hline
\end{tabular}

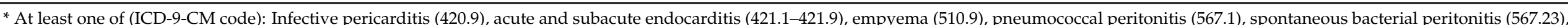

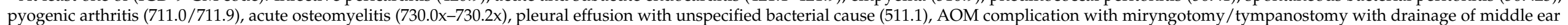
(20.0). AOM: acute otitis media; CI: confidence interval; ICD-9-CM: International Classification of Diseases, Ninth Revision, Clinical Modification; SD, standard deviation 
Table 2. Healthcare resource utilization and costs for all-cause pneumonia for subjects $\geq 15$ years of age in the Liguria region 2012-2018.

\begin{tabular}{|c|c|c|c|c|c|c|c|c|c|}
\hline \multirow{2}{*}{ Year } & \multicolumn{3}{|c|}{$\begin{array}{l}\text { ED Visits (Not Followed } \\
\text { by Hospitalization) }\end{array}$} & \multicolumn{3}{|c|}{ ED Visits + Other Hospitalization } & \multicolumn{3}{|c|}{$\begin{array}{l}\text { Total Access to ED } \\
\text { and Hospitalizations }\end{array}$} \\
\hline & Number & Costs $(€)$ & $\begin{array}{l}\text { Cost per } \\
\text { Event }(€)\end{array}$ & Number & Costs $(€)$ & $\begin{array}{l}\text { Cost per } \\
\text { Event }(€)\end{array}$ & Number & Costs $(€)$ & $\begin{array}{l}\text { Cost per } \\
\text { Event }(€)\end{array}$ \\
\hline 2012 & 1114 & 104,920 & 94.18 & 7210 & $32,028,752$ & 4442 & 8324 & $32,133,672$ & 3860 \\
\hline 2013 & 1210 & 119,713 & 98.94 & 7459 & $32,968,282$ & 4420 & 8669 & $33,087,995$ & 3817 \\
\hline 2014 & 1684 & 169,536 & 100.67 & 7765 & $35,275,129$ & 4543 & 9446 & $35,444,665$ & 3752 \\
\hline 2015 & 1681 & 181,105 & 107.74 & 8689 & $38,705,514$ & 4455 & 10,370 & $38,886,619$ & 3750 \\
\hline 2016 & 1600 & 177,025 & 110.64 & 8436 & $38,416,440$ & 4554 & 10,036 & $38,593,465$ & 3846 \\
\hline 2017 & 1800 & 203,609 & 113.12 & 9326 & $40,576,454$ & 4351 & 11,126 & $40,780,063$ & 3665 \\
\hline 2018 & 2126 & 234,526 & 110.31 & 10,346 & $43,694,624$ & 4223 & 12,472 & $43,929,150$ & 3522 \\
\hline Weighted average & & 168,954 & 106.15 & & $37,845,435$ & 4418 & & $38,291,363$ & 3219 \\
\hline
\end{tabular}

ED: emergency department.

Table 3. Healthcare resource utilization and costs for specified and unspecified pneumonia for individuals $\geq 15$ years of age in the Liguria region 2012-2018.

\begin{tabular}{|c|c|c|c|c|c|c|c|c|c|}
\hline \multirow{2}{*}{ Year } & \multicolumn{3}{|c|}{$\begin{array}{l}\text { ED Visits (Not Followed } \\
\text { by Hospitalization) }\end{array}$} & \multicolumn{3}{|c|}{ ED Visits + Other Hospitalization } & \multicolumn{3}{|c|}{$\begin{array}{l}\text { Total Access to Hospitalization } \\
\text { and ED }\end{array}$} \\
\hline & Number & Costs $(€)$ & $\begin{array}{l}\text { Cost per } \\
\text { Event }(€)\end{array}$ & Number & Costs $(€)$ & $\begin{array}{l}\text { Cost per } \\
\text { Event }(€)\end{array}$ & Number & Costs $(€)$ & $\begin{array}{l}\text { Cost per } \\
\text { Event }(€)\end{array}$ \\
\hline \multicolumn{10}{|c|}{ Pneumococcal pneumonia } \\
\hline 2012 & 0 & 0 & - & 79 & 380,836 & 4821 & 79 & 380,836 & 4821 \\
\hline 2013 & 0 & 0 & - & 121 & 610,601 & 5046 & 121 & 610,601 & 5046 \\
\hline 2014 & 0 & 0 & - & 118 & 611,587 & 5183 & 118 & 611,587 & 5183 \\
\hline 2015 & 0 & 0 & - & 121 & 531,407 & 4392 & 121 & 531,407 & 4392 \\
\hline 2016 & 0 & 0 & - & 107 & 517,943 & 4841 & 107 & 517,943 & 4841 \\
\hline 2017 & 0 & 0 & - & 158 & 784,849 & 4967 & 158 & 784,849 & 4967 \\
\hline 2018 & 0 & 0 & - & 169 & 754,212 & 4463 & 169 & 754,212 & 4463 \\
\hline Weighted average & & & & & 626,946 & 4801 & & 626,946 & 4801 \\
\hline \multicolumn{10}{|c|}{ Unspecified pneumonia } \\
\hline 2012 & 173 & 16,931 & 98 & 6629 & $23,302,741$ & 3515 & 6802 & $23,319,672$ & 3428 \\
\hline 2013 & 163 & 16,397 & 101 & 6925 & $24,237,574$ & 3500 & 7088 & $24,253,971$ & 3422 \\
\hline 2014 & 165 & 18,164 & 110 & 7555 & $26,495,484$ & 3507 & 7720 & $26,513,648$ & 3434 \\
\hline 2015 & 197 & 25,343 & 129 & 8310 & $29,987,075$ & 3609 & 8507 & $30,012,418$ & 3528 \\
\hline 2016 & 226 & 27,615 & 122 & 8047 & $29,835,985$ & 3708 & 8273 & $29,863,600$ & 3610 \\
\hline 2017 & 213 & 25,229 & 118 & 8974 & $32,355,936$ & 3606 & 9187 & $32,381,165$ & 3525 \\
\hline 2018 & 244 & 27,590 & 113 & 9878 & $34,706,579$ & 3514 & 10,122 & $34,734,169$ & 3432 \\
\hline Weighted average & & 18,740 & 114 & & $29,208,583$ & 3568 & & $29,232,052$ & 3485 \\
\hline
\end{tabular}

Table 4. Healthcare resource utilization and costs for meningitis for individuals $\geq 15$ years of age in the Liguria region 2012-2018.

\begin{tabular}{|c|c|c|c|c|c|c|c|}
\hline \multirow[b]{2}{*}{ Year } & \multicolumn{4}{|c|}{ ED Visits + Other Hospitalization } & \multicolumn{3}{|c|}{ Total Access to Hospitalization and ED } \\
\hline & Number & Number & Costs $(€)$ & $\begin{array}{l}\text { Cost per } \\
\text { Event }(€)\end{array}$ & Number & Costs $(€)$ & $\begin{array}{l}\text { Cost per } \\
\text { Event }(€)\end{array}$ \\
\hline \multicolumn{8}{|c|}{ Pneumococcal meningitis } \\
\hline 2012 & 0 & 12 & 79,267 & 6606 & 12 & 79,267 & 6606 \\
\hline 2013 & 0 & 15 & 147,537 & 9836 & 15 & 147,537 & 9836 \\
\hline 2014 & 0 & 10 & 55,286 & 5529 & 10 & 55,286 & 5529 \\
\hline 2015 & 0 & 20 & 260,327 & 13,016 & 20 & 260,327 & 13,016 \\
\hline 2016 & 0 & 18 & 144,186 & 8010 & 18 & 144,186 & 8010 \\
\hline 2017 & 0 & 23 & 262,049 & 11,393 & 23 & 262,049 & 11,393 \\
\hline 2018 & 0 & 19 & 175,319 & 9227 & 19 & 175,319 & 9227 \\
\hline
\end{tabular}


Table 4. Cont.

\begin{tabular}{|c|c|c|c|c|c|c|c|}
\hline \multirow[b]{2}{*}{ Year } & \multicolumn{4}{|c|}{ ED Visits + Other Hospitalization } & \multicolumn{3}{|c|}{ Total Access to Hospitalization and ED } \\
\hline & Number & Number & Costs $(€)$ & $\begin{array}{l}\text { Cost per } \\
\text { Event }(€)\end{array}$ & Number & Costs $(€)$ & $\begin{array}{l}\text { Cost per } \\
\text { Event }(€)\end{array}$ \\
\hline Weighted average & & & 178,438 & 9606 & & 178,438 & 9606 \\
\hline \multicolumn{8}{|c|}{ Unspecified meningitis } \\
\hline 2012 & 0 & 10 & 104,321 & 10,432 & 10 & 104,321 & 10,432 \\
\hline 2013 & 1 & 4 & 78,047 & 19,512 & 5 & 78,347 & 15,669 \\
\hline 2014 & 0 & 8 & 50,587 & 6323 & 8 & 50,587 & 6323 \\
\hline 2015 & 1 & 10 & 113,323 & 11,332 & 11 & 113,384 & 10,308 \\
\hline 2016 & 0 & 9 & 66,686 & 7410 & 9 & 66,686 & 7410 \\
\hline 2017 & 0 & 6 & 40,988 & 6831 & 6 & 40,988 & 6831 \\
\hline 2018 & 0 & 10 & 93,125 & 9313 & 10 & 93,125 & 9313 \\
\hline Weighted average & & & 81,942 & 9598 & & 82,444 & 9279 \\
\hline
\end{tabular}

ED: emergency department.

Table 5. Healthcare resource utilization and costs for bacteremia for individuals $\geq 15$ years of age in the Liguria region 2012-2018.

\begin{tabular}{|c|c|c|c|c|c|c|}
\hline \multirow[b]{2}{*}{ Year } & \multicolumn{3}{|c|}{ ED Visits + Other Hospitalization } & \multicolumn{3}{|c|}{ Total Access to Hospitalization and ED } \\
\hline & Number & Costs $(€)$ & $\begin{array}{l}\text { Cost per } \\
\text { Event }(€)\end{array}$ & Number & Costs $(€)$ & $\begin{array}{l}\text { Cost per } \\
\text { Event }(€)\end{array}$ \\
\hline \multicolumn{7}{|c|}{ Pneumococcal bacteremia } \\
\hline 2012 & 85 & 550,487 & 6476 & 85 & 550,487 & 6476 \\
\hline 2013 & 114 & 840,435 & 7372 & 114 & 840,435 & 7372 \\
\hline 2014 & 129 & 783,265 & 6072 & 129 & 783,265 & 6072 \\
\hline 2015 & 134 & 921,595 & 6878 & 134 & 921,595 & 6878 \\
\hline 2016 & 166 & $1,291,753$ & 7782 & 166 & $1,291,753$ & 7782 \\
\hline 2017 & 171 & $1,431,004$ & 8368 & 171 & $1,431,004$ & 8368 \\
\hline 2018 & 149 & $1,250,853$ & 8395 & 149 & $1,250,853$ & 8395 \\
\hline Weighted average & & $1,068,192$ & 7457 & & $1,068,192$ & 7457 \\
\hline \multicolumn{7}{|c|}{ Unspecified bacteremia } \\
\hline 2012 & 951 & $5,248,597$ & 5519 & 954 & $5,249,230$ & 5502 \\
\hline 2013 & 979 & $5,202,355$ & 5314 & 979 & $5,202,355$ & 5314 \\
\hline 2014 & 1116 & $6,459,316$ & 5788 & 1116 & $6,459,316$ & 5788 \\
\hline 2015 & 1244 & $7,983,688$ & 6418 & 1244 & $7,983,688$ & 6418 \\
\hline 2016 & 1252 & $8,627,310$ & 6891 & 1252 & $8,627,310$ & 6891 \\
\hline 2017 & 1224 & $8,205,921$ & 6704 & 1225 & $8,206,221$ & 6699 \\
\hline 2018 & 1221 & $8,064,550$ & 6605 & 1223 & $8,065,150$ & 6595 \\
\hline Weighted average & & $7,362,056$ & 6283 & & $7,362,261$ & 6279 \\
\hline
\end{tabular}

ED: emergency department; NR: not reported.

\subsection{Prescribed Medication during Follow-Up}

The number of pharmaceutical prescriptions, days of drug therapy, and associated costs are shown in Table 6. The median annual number of pharmaceutical prescriptions in the follow-up period was 13,541 , with a median annual cost of $€ 7,997,000$, including $€ 340,140$ for antibacterial agents.

Table 6. Pharmaceutical and antibiotics prescriptions costs during the 6-month follow-up period for individuals $\geq 15$ years of age in the Liguria region 2012-2018.

\begin{tabular}{|c|c|c|c|c|c|c|c|c|c|}
\hline \multirow[b]{2}{*}{ Year } & \multicolumn{3}{|c|}{ Pharmaceutical Prescriptions } & \multicolumn{3}{|c|}{ Antimicrobials } & \multicolumn{3}{|c|}{ Antibacterials } \\
\hline & Number & $\begin{array}{l}\text { Days of Drug } \\
\text { Therapy }\end{array}$ & Costs $(€)$ & Number & $\begin{array}{l}\text { Days of Drug } \\
\text { Therapy }\end{array}$ & Costs $(€)$ & Number & $\begin{array}{c}\text { Days of Drug } \\
\text { Therapy }\end{array}$ & Costs $(€)$ \\
\hline 2012 & 11,912 & $4,036,659$ & $5,814,942$ & 6640 & 131,825 & 713,788 & 6481 & 98,933 & 339,138 \\
\hline 2013 & 12,206 & $4,225,424$ & $6,313,103$ & 6753 & 135,076 & 791,291 & 6591 & 103,664 & 372,499 \\
\hline
\end{tabular}


Table 6. Cont.

\begin{tabular}{|c|c|c|c|c|c|c|c|c|c|}
\hline \multirow[b]{2}{*}{ Year } & \multicolumn{3}{|c|}{ Pharmaceutical Prescriptions } & \multicolumn{3}{|c|}{ Antimicrobials } & \multicolumn{3}{|c|}{ Antibacterials } \\
\hline & Number & $\begin{array}{c}\text { Days of Drug } \\
\text { Therapy }\end{array}$ & Costs $(€)$ & Number & $\begin{array}{c}\text { Days of Drug } \\
\text { Therapy }\end{array}$ & Costs $(€)$ & Number & $\begin{array}{c}\text { Days of Drug } \\
\text { Therapy }\end{array}$ & Costs $(€)$ \\
\hline 2014 & 12,837 & $4,662,019$ & $6,740,202$ & 7361 & 142,900 & 813,195 & 7209 & 110,989 & 370,273 \\
\hline 2015 & 13,675 & $5,237,878$ & $7,996,969$ & 7553 & 155,272 & $1,000,425$ & 7397 & 118,067 & 330,834 \\
\hline 2016 & 13,541 & $5,457,865$ & $8,800,113$ & 7457 & 158,361 & $1,522,924$ & 7284 & 120,098 & 340,142 \\
\hline 2017 & 14,583 & $6,265,637$ & $8,802,385$ & 7906 & 172,000 & $1,169,078$ & 7743 & 132,478 & 312,532 \\
\hline 2018 & 15,393 & $7,343,894$ & $11,010,118$ & 8372 & 171,744 & $1,294,140$ & 8182 & 135,981 & 340,541 \\
\hline \multicolumn{3}{|c|}{ Median annual cost } & $7,996,969$ & & & $1,000,425$ & & & 340,140 \\
\hline
\end{tabular}

\section{Discussion}

This study has shown that, despite routine vaccination of children, there are still substantial economic costs associated with all-cause pneumonia, pneumococcal and unspecified pneumonia, bacteremia, meningitis, and AOM in individuals $\geq 15$ years of age in the Liguria region of Italy. The Ligurian infant immunization program has achieved high levels of vaccine coverage $(>90 \%)$ since 2007 . However, this study shows that the economic impact of PD on adults remains high, with an annual cost of $€ 48.97$ million during the study period. Furthermore, immunization programs addressed to adult high-risk populations and the elderly are also implemented with unsatisfactory vaccination coverages.

In our study, all-cause pneumonia and pneumococcal and unspecified pneumonia accounted for the majority of hospitalization costs during the study. The median annual cost of hospitalization for all-cause pneumonia was $€ 38,416,440$ (per-capita cost: $€ 26.78$ ) and $€ 30,353,928$ (per-capita cost: $€ 20.88$ ) for pneumococcal pneumonia and unspecified pneumonia. Bacteremia and meningitis accounted for a lower proportion of costs of ED visits and hospitalizations. The costs associated with ED visits and hospitalizations due to PD also generally increased during the study period from 2012 to 2018.

A substantial healthcare utilization cost due to PD was reported in Italy as a whole, as well as in other countries. In Italy, costs related to PD have increased regardless of the introduction of PCVs in infants. In Italian adults $>64$ years of age, hospitalization costs for PD increased by $33.8 \%$ after the introduction of PCV7/PCV13 [23]. In Spain, the estimated cost of hospitalizations in 2011 in adults $\geq 18$ years of age was more than $€ 57$ million for all PD, and $€ 47$ million for pneumococcal pneumonia [24]. In the United States in 2015, total costs associated with PD in adults $\geq 19$ years of age were US $\$ 1.86$ billion, of which US $\$ 1.8$ billion was attributable to direct inpatient, outpatient, and medication costs [25]. PD-related costs are higher in adults with high-risk conditions and in those with IPD [24,26], and our study certainly reports a higher level of comorbidities in the study population compared with the general Ligurian adult population.

A key strength of the study is that it assessed episodes of PD as well as disease of unknown etiology. As such, it provided both a highly specific estimate of cost, with a high degree of certainty that episodes included in the analysis were caused by S. pneumoniae, and one that is more sensitive, with the potential to capture additional undetected pneumococcal episodes and those potentially caused by other pathogens. However, there are also some methodological limitations that must be considered. Firstly, the presence of a diagnosis code does not necessarily indicate the presence of disease, due to the possibility of incorrect coding.

In fact, analysing a total of 70,402 records occurring in Liguria from 2012-2018 with at least one ICD-9 CM diagnosis code suggestive of all-cause pneumonia, only $1.37 \%$ hospitalizations had specific ICD-9 CM diagnosis codes for pneumococcal pneumonia. This apparent low impact can be related to the available data on hospital discharge records in Italy, which are subject to some limitations (common to all passive surveillance systems), such as underestimations or deficiencies in reporting, due especially to the frequent lack of etiological identification of the causal agent during the medical care of the disease.

As a consequence, the etiologic fraction attributable to S. pneumoniae may be underdiagnosed or, alternatively, overestimated, considering all potentially attributable pneumococcal diseases in the absence of microbiological identification. 
Accordingly, several studies have reported that ICD-9 CM codes suggest that pneumococcal pneumonia have good specificity, but poor sensitivity $[27,28]$. Thus, our results represent a good starting point to reinforce strategies addressed to improve more sensitive surveillance systems that are needed nationwide. Hospitals in particular, where more severe cases are observed, could be one of the key places where a specific surveillance program can be performed with the most updated techniques of molecular biology.

Secondly, indirect costs, such as productivity loss, the cost of transportation, and loss of earnings, were not taken into account.

Finally, in our study, we used ICD-9-CM codification, although the standard is now at its eleventh revision (ICD-11), approved in 2018 by the Member States, at the 72 World Health Assembly. The decision to use the old coding system could suggest some inaccuracies in our data, but in Italy the new version mentioned above will come into force on 1 January 2022.

ICD-9-CM is the version of ICD currently utilised in Italy, according to legislation, for the coding of diseases and related problems, for statistical studies on morbidity and mortality rates, and for epidemiological studies. It is also recognized as a valid management tool for public health and hygiene [29].

\section{Conclusions}

Despite widespread pediatric and older adult vaccination programs in Liguria, PD in adults continues to incur high economic costs due to ED visits and hospitalizations. In this study, the majority of costs were due to all-cause, pneumococcal-specific, and unspecified pneumonia. The results of this study confirm the need to reinforce vaccination programs in adults at risk, especially those with comorbidities.

Supplementary Materials: The following are available online at https://www.mdpi.com/article/10.3 390/vaccines9121380/s1, Table S1: Search terms and ICD-9 codes of pneumonia.

Author Contributions: Conceptualization, F.A., A.D., F.S., G.M.P. and G.I.; methodology, M.A., C.S., F.A., L.S. and A.O.; validation, C.P., C.T., M.F.P. and F.A.; formal analysis, M.A. and F.T.; data curation, M.F.P., B.D.S., D.A., C.T., C.S. and A.O.; writing-original draft preparation, review and editing, A.D., F.S., G.M.P., F.A., C.P., D.A., M.A., M.F.P., C.T., A.O. and G.I.; supervision, G.I. and F.A. All authors have read and agreed to the published version of the manuscript.

Funding: Funding was provided by Merck Sharp \& Dohme Corp., a subsidiary of Merck \& Co., Inc., Kenilworth, NJ, USA.

Institutional Review Board Statement: Data collected in the Administrative healthcare data Regional Service are transmitted by the Ligurian Local Health Units and hospitals to the regional health agency of Liguria "Azienda Ligure Sanitaria" (A.Li.Sa.). Thus, ethical review and approval were waived for this study because the institutional activities of A.Li.Sa. include handling regional healthcare administrative data and conduct epidemiological studies, projects and researches to support strategical choices of healthcare [30]. The current regulation on privacy allows professionals belonging to the regional A.Li.Sa. accessing to healthcare administrative data routinely transmitted by the public funded LHUs and hospitals of the Liguria region. Data are anonymous and have been evaluated in an aggregate manner. Finally, patients who access to regional public health services give consent to the use of healthcare data also for scientific purpose.

Informed Consent Statement: Administrative healthcare data or Data Warehouse, is a regional service that collects Hospital Discharge Records (HDRs), the flow of outpatient visits, and pharmaceutical and was used as a data source. HDR data are recorded with the consent of the patient and can be used for scientific studies in the form of aggregated and de-identified data.

Data Availability Statement: Merck Sharp \& Dohme Corp., a subsidiary of Merck \& Co., Inc., Kenilworth, NJ, USA's data sharing policy, including restrictions, is available at http:/ / engagezone. msd.com/ds_documentation.php (accessed on 9 November 2021) through the EngageZone site or via email to dataaccess@merck.com. 
Acknowledgments: We thank Sarah Amir, who provided writing support and Ian Norton, who provided editorial support prior to submission on behalf of Scion, London, UK, supported by Merck Sharp \& Dohme Corp., a subsidiary of Merck \& Co., Inc., Kenilworth, NJ, USA, according to Good Publication Practice guidelines (Link). The Sponsor was involved in the study design, collection, analysis, and interpretation of data, as well as data checking of information provided in the manuscript. However, ultimate responsibility for opinions, conclusions, and data interpretation lies with the authors.

Conflicts of Interest: A.D., F.S. and G.M.P. are employees of Merck Sharp \& Dohme Corp., a subsidiary of Merck \& Co., Inc., Kenilworth, NJ, USA, and MSD, Rome, Italy, and may own stock and/or stock options in Merck \& Co., Inc., Kenilworth, NJ, USA. G.I. and A.O. received grants/contracts from MSD, GSK, Sanofi Pasteur, Pfizer, Seqirus, and Janssen, and support for attending meetings/travel from MSD, GSK, Sanofi Pasteur, Pfizer, and Seqirus. L.S. received grants/contracts from MSD, GSK, Sanofi Pasteur, Pfizer, Seqirus, AJVaccines and Janssen, and support for attending meetings/travel from MSD, GSK, Sanofi Pasteur, Pfizer, and Seqirus. F.A. received funding/support from MSD. M.A., B.D.S., M.F.P., D.A., F.T., C.T., C.P. and C.S. have no conflict of interest.

\section{References}

1. Centers for Disease Control and Prevention. Pneumococcal Disease-Types of Infection. 2017. Available online: https://www.cdc. gov/pneumococcal/about/infection-types.html (accessed on 22 September 2021).

2. Tan, T.Q. Pediatric invasive pneumococcal disease in the United States in the era of pneumococcal conjugate vaccines. Clin. Microbiol. Rev. 2012, 25, 409-419. [CrossRef]

3. GBD 2016 Lower Respiratory Infections Collaborators. Estimates of the global, regional, and national morbidity, mortality, and aetiologies of lower respiratory infections in 195 countries, 1990-2016: A systematic analysis for the Global Burden of Disease Study 2016. Lancet Infect Dis. 2018, 18, 1191-1210. [CrossRef]

4. Huang, S.S.; Johnson, K.M.; Ray, G.T.; Wroe, P.; Lieu, T.A.; Moore, M.R.; Zell, E.R.; Linder, J.; Grijalva, C.G.; Metlay, J.P.; et al. Healthcare utilization and cost of pneumococcal disease in the United States. Vaccine 2011, 29, 3398-3412. [CrossRef] [PubMed]

5. Song, J.Y.; Choi, J.Y.; Lee, J.S.; Bae, I.-G.; Kim, Y.K.; Sohn, J.W.; Jo, Y.M.; Choi, W.S.; Lee, J.; Park, K.H.; et al. Clinical and economic burden of invasive pneumococcal disease in adults: A multicenter hospital-based study. BMC Infect Dis. 2013, 13, 202. [CrossRef] [PubMed]

6. Weinberger, D.M.; Harboe, Z.B.; Shapiro, E.D. Developing better pneumococcal vaccines for adults. JAMA Intern. Med. 2017, 177, 303-304. [CrossRef]

7. Weinberger, D.M.; Grant, L.R.; Weatherholtz, R.C.; Warren, J.L.; O’Brien, K.L.; Hammitt, L.L. Relating Pneumococcal Carriage Among Children to Disease Rates Among Adults Before and After the Introduction of Conjugate Vaccines. Am. J. Epidemiol. 2016, 183, 1055-1062. [CrossRef] [PubMed]

8. $\quad$ van de Garde, M.D.; Knol, M.J.; Rots, N.Y.; van Baarle, D.; van Els, C.A. Vaccines to Protect Older Adults against Pneumococcal Disease. Interdiscip. Top. Gerontol. Geriatr. 2020, 43, 113-130.

9. Georgalis, L.; Mozalevskis, A.; De Aragón, M.M.; Garrido-Estepa, M. Changes in the pneumococcal disease-related hospitalisations in Spain after the replacement of 7-valent by 13-valent conjugate vaccine. Eur. J. Clin. Microbiol. Infect. Dis. 2017, 36, 575-583. [CrossRef] [PubMed]

10. Durando, P.; Crovari, P.; Ansaldi, F.; Sticchi, L.; Sticchi, C.; Turello, V.; Marensi, L.; Giacchino, R.; Timitilli, A.; Carloni, R.; et al. Universal childhood immunisation against Streptococcus pneumoniae: The five-year experience of Liguria Region, Italy. Vaccine 2009, 27, 3459-3462. [CrossRef]

11. Orsi, A.; Ansaldi, F.; Trucchi, C.; Rosselli, R.; Icardi, G. Pneumococcus and the elderly in Italy: A summary of available evidence regarding carriage, clinical burden of lower respiratory tract infections and on-field effectiveness of PCV13 vaccination. Int. J. Mol. Sci. 2016, 17, 1140. [CrossRef]

12. Ansaldi, F.; Sticchi, L.; Durando, P.; Carloni, R.; Oreste, P.; Vercelli, M.; Crovari, P.; Icardi, G. Decline in pneumonia and acute otitis media after the introduction of childhood pneumococcal vaccination in Liguria, Italy. J. Int. Med. Res. 2008, 36, 1255-1260. [CrossRef]

13. Ministero della Salute. DGPRV 0024720-P-27/05/2010. Indicazioni in Merito alla Somministrazione del Vaccino Antipneumococcico Prevenar 13 in età Pediatrica. 2010. Available online: https://www.fimpcalabria.org/public/vaccinazioni/indicazioni\%20 in $\% 20$ merito $\% 20$ alla\%20somministrazione \%20del\%20vaccino $\% 20$ antipneumococcico $\% 20$ prevenar $\% 2013 \% 20 \mathrm{in} \% 20 \mathrm{et} \%$ C3 $\%$ A0 \%20pediatrica\%20(2).pdf (accessed on 22 September 2021).

14. Ansaldi, F.; De Florentis, D.; Canepa, P.; Bandettini, R.; Diana, M.C.; Martini, M.; Durando, P.; Icardi, G. Epidemiological changes after PCV7 implementation in Italy: Perspective for new vaccines. Hum. Vaccin. 2011, 7, 211-216. [CrossRef] [PubMed]

15. EpiCentro Epidemiology for Public Health. Vaccinations in Italy. Available online: https://www.epicentro.iss.it/vaccini/dati_Ita (accessed on 22 September 2021).

16. Orsi, A.; Ansaldi, F.; Durando, P.; Turello, V.; Icardi, G. Immunization campaign with 13-valent Pneumococcal Conjugate Vaccine in adults in Liguria Region, Italy: One year post-introduction preliminary results. Epidemiol. Prev. 2014, 38 (Suppl. 2), 66-72. 
17. Chalmers, J.; Campling, J.; Ellsbury, G.; Hawkey, P.M.; Madhava, H.; Slack, M. Community-acquired pneumonia in the United Kingdom: A call to action. Pneumonia (Nathan) 2017, 9, 15. [CrossRef]

18. Torres, A.; Cillóniz, C.; Blasi, F.; Chalmers, J.D.; Gaillat, J.; Dartois, N.; Schmitt, H.-J.; Welte, T. Burden of pneumococcal community-acquired pneumonia in adults across Europe: A literature review. Respir. Med. 2018, 137, 6-13. [CrossRef] [PubMed]

19. Monali, R.; De Vita, E.; Mariottini, F.; Privitera, G.; Lopalco, P.L.; Tavoschi, L. Impact of vaccination on invasive pneumococcal disease in Italy 2007-2017: Surveillance challenges and epidemiological changes. Epidemiol. Infect. 2020, 148, e187. [CrossRef] [PubMed]

20. Navarro-Torné, A.; Montuori, E.A.; Kossyvaki, V.; Méndez, C. Burden of pneumococcal disease among adults in Southern Europe (Spain, Portugal, Italy, and Greece): A systematic review and meta-analysis. Hum. Vaccines Immunother. 2021, 17, 3670-3686. [CrossRef]

21. Trucchi, C.; Paganino, C.; Orsi, A.; Amicizia, D.; Tisa, V.; Piazza, M.F.; Gallo, D.; Simonetti, S.; Buonopane, B.; Icardi, G.; et al. Hospital and economic burden of influenza-like illness and lower respiratory tract infection in adults $\geq 50$ years-old. BMC Health Serv. Res. 2019, 19, 1-11. [CrossRef] [PubMed]

22. Ministero della Salute. Supplemento Ordinario n. 8 alla GAZZETTA UFFICIALE 28.01.2013-Serie Generale-n. 23. 2013. Available online: https: / / www.trovanorme.salute.gov.it/norme/renderPdf.spring?seriegu=SG\&datagu=28/01/2013\&redaz=13A00528 \&artp=1\&art=1\&subart=1\&subart1=10\&vers=1\&prog=001 (accessed on 22 September 2021).

23. Boccalini, S.; Varone, O.; Chellini, M.; Pieri, L.; Sala, A.; Berardi, C.; Bonanni, P.; Bechini, A. Hospitalizations for pneumonia, invasive diseases and otitis in Tuscany (Italy), 2002-2014: Which was the impact of universal pneumococcal pediatric vaccination? Hum. Vaccin. Immunother. 2017, 13, 428-434. [CrossRef]

24. Gil-Prieto, R.; Pascual-Garcia, R.; Walter, S.; Álvaro-Meca, A.; Gil-De-Miguel, Á. Risk of hospitalization due to pneumococcal disease in adults in Spain. The CORIENNE study. Hum. Vaccin. Immunother. 2016, 12, 1900-1905. [CrossRef]

25. Ozawa, S.; Portnoy, A.; Getaneh, H.; Clark, S.; Knoll, M.D.; Bishai, D.; Yang, H.K.; Patwardhan, P.D. Modeling the economic burden of adult vaccine-preventable diseases in the United States. Health Aff. (Millwood) 2016, 35, 2124-2132. [CrossRef] [PubMed]

26. Weycker, D.; Farkouh, R.A.; Strutton, D.R.; Edelsberg, J.; Shea, K.M.; Pelton, S.I. Rates and costs of invasive pneumococcal disease and pneumonia in persons with underlying medical conditions. BMC Health Serv. Res. 2016, 16, 182. [CrossRef] [PubMed]

27. Guevara, R.E.; Butler, J.C.; Marston, B.J.; Plouffe, J.F.; File, T.M.; Breiman, R.F. Accuracy of ICD-9-CM codes in detecting community-acquired pneumococcal pneumonia for incidence and vaccine efficacy studies. Am. J. Epidemiol. 1999, 149, 282-289. [CrossRef] [PubMed]

28. van de Garde, E.M.; Oosterheert, J.J.; Bonten, M.; Kaplan, R.C.; Leufkens, H.G. International classification of diseases codes showed modest sensitivity for detecting community-acquired pneumonia. J. Clin. Epidemiol. 2007, 60, 834-838. [CrossRef]

29. Agenzia per 1'Italia Digitale. Fascicolo Sanitario Elettronico ICD-9-CM 5 August 2021. Available online: https://www. fascicolosanitario.gov.it/en/ICD-9-CM (accessed on 9 November 2021).

30. Regional Law 29/07/2016, n.17. Istituzione dell'Azienda Ligure Sanitaria della Regione Liguria (A.Li.Sa.) e Indirizzi per il Riordino delle Disposizioni Regionali in Materia Sanitaria e Sociosanitaria. Bollettino Ufficiale n.15, 30/07/2016. Available online: http:/ / lrv.regione.liguria.it/liguriass_prod/articolo?urndoc=urn:nir:regione.liguria:legge:2016-07-29;17\&pr=idx,0;artic, 1; articparziale,0 (accessed on 22 November 2021). 